\title{
Curative embolization of a vein of Galen malformation with a dual-lumen balloon and ethylene vinyl alcohol copolymer
}

\author{
Luís Henrique de Castro-Afonso ${ }^{1}$; Daniel Giansante Abud $^{1}$
}

\begin{abstract}
${ }^{1}$ Division of Interventional Neuroradiology, Department of Medical Imaging, Hematology and Oncology, Ribeirão Preto Medical School, University of São Paulo, Ribeirão Preto, SP, Brazil.
\end{abstract}

To whom correspondence should be addressed: Daniel Giansante Abud, MD, PhD e-mail:dgabud@gmail.com

Available at:

http://www.archpedneurosurg.com.br/

\begin{abstract}
The endovascular approach is the standard treatment of vein of Galen malformations (VOGMs,) however the morbidity and mortality rates of VOGMs treated by embolization are still significant. Embolization with ethylene vinyl alcohol copolymer (EVOH) by a dual-lumen balloon (DLB) is a promising. In the present case we demonstrated two strategies of transarterial (TA) embolization of a mural type VOGM presenting with two direct shunts. The first shunt was embolized using coils to reduce the flow through the shunt, following by an EVOH injection which resulted in the complete occlusion of that shunt. The second shunt was embolized also with EVOH but through a DLB. In the second embolization a very precise cast of $\mathrm{EVOH}$ could be created resulting in a complete occlusion of the VOGM. This case demonstrated the advantages of DLB compared to other embolization techniques.
\end{abstract}

\section{INTRODUCTION}

Vein of Galen malformations (VOGMs) are rare congenital cerebrovascular conditions. VGOMs represents around $1 \%$ of all intracranial vascular lesions in the general population, while account for $30 \%$ of the intracranial vascular malformations in the pediatric population $(1,2)$. Despite the endovascular treatment (EVT) is the standard treatment of VOGMs, the morbidity and mortality rates of VOGMs treated by embolization are still significant (3). The reasons for the relatively high rates of poor outcomes of VOGMs are associated with the clinical severity of patients at presentation, but also with technical issues related to the treatment (1-3). Improvements in embolization techniques and embolic materials might increase the curative rates while reduce complications (4-6). Embolization with ethylene vinyl alcohol copolymer (EVOH) by a dual-lumen balloon (DLB) is a promising strategy. The aim of this article was to report effectiveness of the embolization using EVOH through a dual-lumen balloon in the treatment of a mural type VOGM.

\section{CASE REPORT}

A 1.5 years-old boy was admitted presenting with seizures, developmental delay and hydrocephalus. A mural type VOGM was diagnosed. The endovascular treatment was performed in two stages, both by femoral artery puncture and transarterial technique. In the first procedure, a microcatheter (Echelon 10, Medtronic) was navigated through a wire (Traxcess 0.014, Microvention) and positioned in the anterior choroidal artery branch of the shunt. The arterial branch was coiled (Axium, Medtronic) followed by injection of $1.2 \mathrm{~mL}$ of EVOH (Onyx 18, Medtronic) (Figure 1). A second shunt of the right posterior choroidal artery remained visualized in the angiography of the vertebral artery after the first embolization (Figure 1). 


\section{CURATIVE EMBOLIZATION OF A VEIN OF GALEN MALFORMATION WITH A DUAL-LUMEN BALLOON AND ETHYLENE VINYL ALCOHOL COPOLYMER}
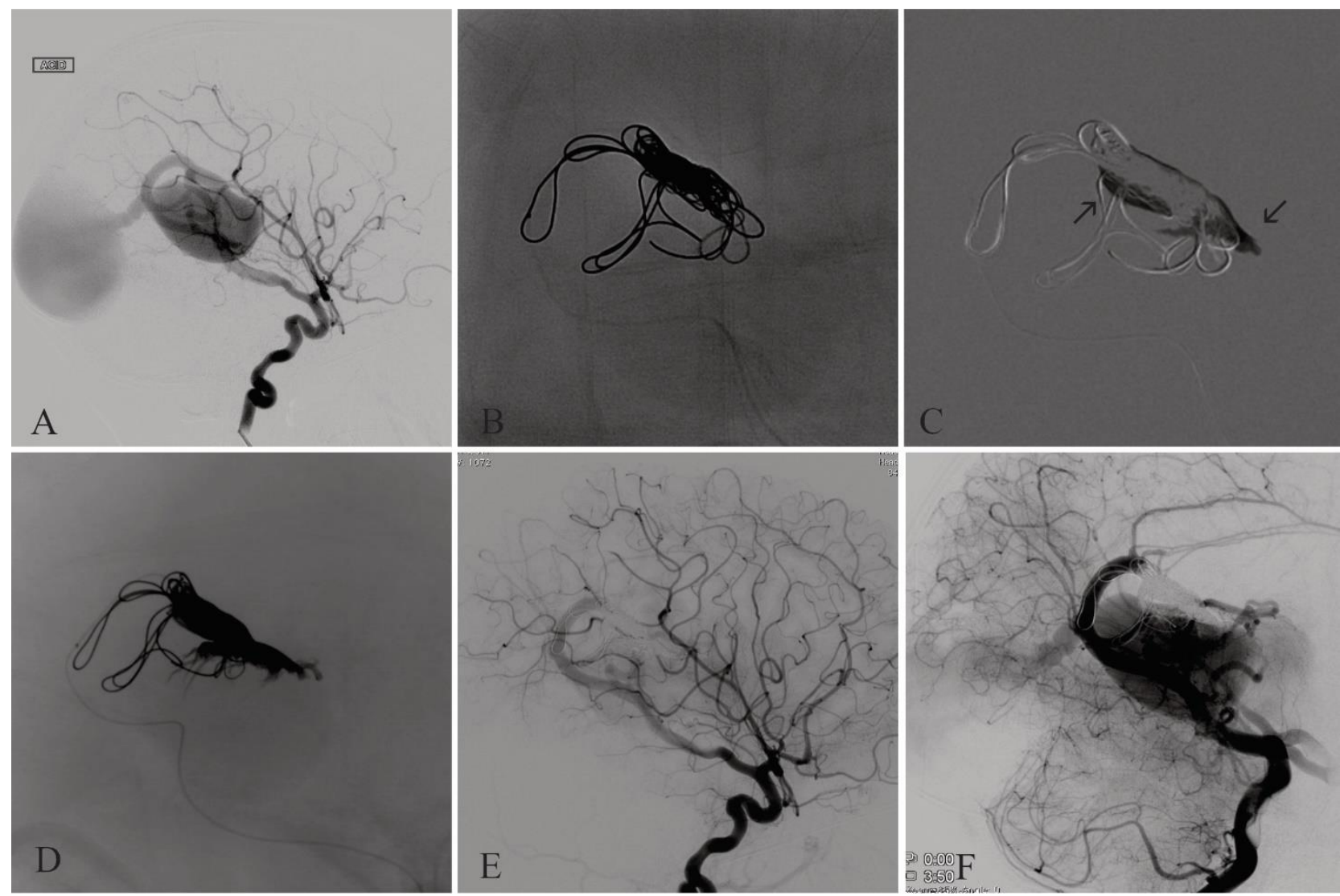

Figure 1 - (A) Digital subtracted angiography (DSA) lateral view of the right internal carotid injection showing a mural type Vein of Galen malformation nourished by the anterior choroidal artery, (B) cast of coils in the anterior choroidal branch to the VGAM, (C) Roadmap lateral view showing an injection of EVOH (Onyx-Medtronic) through the cast of coils, (D) X-ray of the head lateral view showing the final cast of coils and Onyx forming a "mushroom like" shape, (E) DSA lateral view of the right internal carotid artery showing occlusion of the anterior choroidal branch, (F) DSA lateral view of the left vertebral artery showing a second mural shunt of the posterior choroidal artery to the VGAM.

After 3 months, the remnant shunt was occluded in a second embolization session. A DLB (Scepter $4 \times 11 \mathrm{~mm}$, Microvention) was navigated with a wire (Traxcess $0.014-$ inch, Microvention) and placed in the right posterior choroidal arterial branch of the remnant shunt. The balloon was inflated occluding the arterial branch and $2.1 \mathrm{~mL}$ of EVOH (Onyx 18, Medtronic) was injected (Figure 2). The final angiography showed a complete occlusion of the VOGM, and no complications were observed. The patient was discharge after 4 days. During the 12 months follow-up the patient had a progressive improvement in their neurodevelopment status. The figure 3 depicts a computed tomography scan of the brain (CT) at admission showing a dilatation of the median prosencephalic vein of Markowski, falcine sinus and torcula (Figure $3, A$ and $B$ ). The figure 3 ( $C$ and $D$ ) depicts the magnetic resonance image at the 6-month follow-up after embolization showing regression of the vein and torcula. The falcine sinus remained preserved draining normal deep structures of the posterior fossa.

\section{DISCUSSION}

Since the 1980 decade when the neuroendovascular technique became the standard treatment of
VOGMs, more than seven hundred patients with VOGM treated by embolization were reported in the literature (1, 2 ). Most of the studies were case series and patients were recently pooled and analyzed $(1,2)$. Of all patients with VOGM treated in the literature $44 \%$ were newborns $(<1$ month), $41 \%$ infants ( $>1$ months and $<2$ years) and only $12 \%$ children ( $>2$ years) or adults. The rates of complete and partial occlusions reported were $57 \%$ and $43 \%$, respectively. While the rates of poor outcomes and mortality after embolization of VOGMs were $31 \%$ and $12 \%$, respectively (2). However, among the patients with VOGM that were not treated the mortality rate was impressive $47 \%$, four times higher than the mortality rates after embolization.

A good clinical outcome defined as absence of neurologic deficits, or mild neurologic impairments that requires little support in daily life, was reported to be achieved in $68 \%$ of patients during a mean follow-up time of 63 months (2). More interesting, the main outcomes of patients with VOGM after embolization had progressive improvements along a timeline. The good clinical outcome rate of embolized patients over the 1980s, 1990s, and 2000s was 49\%, 70\%, and $70 \%$, respectively, while the poor clinical outcome rate of embolized patients over the 1980s, 1990s, and 2000s was 


\section{CURATIVE EMBOLIZATION OF A VEIN OF GALEN MALFORMATION WITH A DUAL-LUMEN BALLOON AND ETHYLENE VINYL ALCOHOL COPOLYMER}

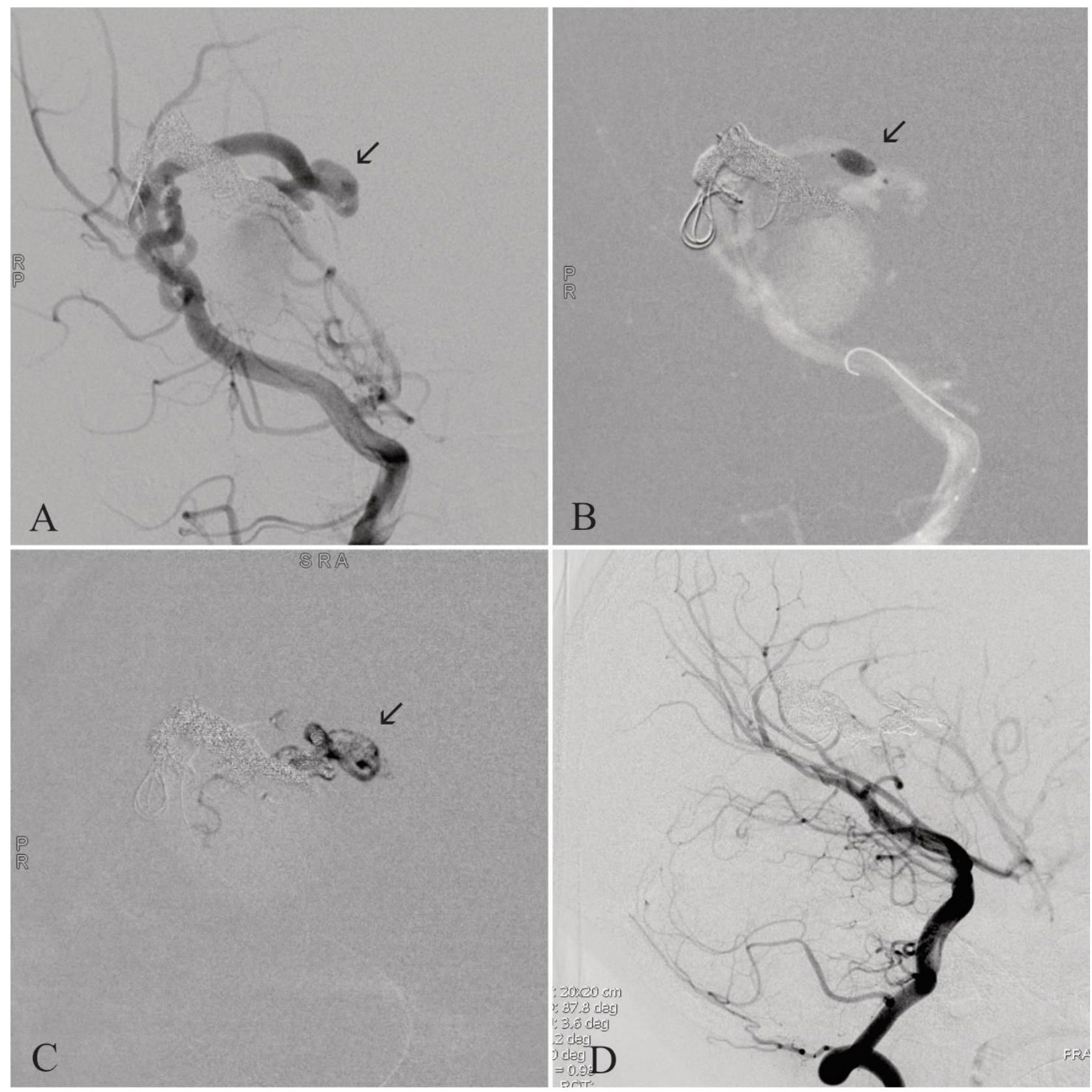

Figure 2 - (A) DSA lateral view of the left vertebral artery showing the remnant mural shunt of the posterior choroidal artery to the VGOM, (B) Roadmap lateral view showing the DLB (black arrow) inflated and occluding the posterior choroidal branch, (C) Roadmap lateral view showing the injection of EVOH (black arrow) through the inflated DLB, (D) DSA lateral view of the left vertebral artery showing a complete occlusion of the posterior choroidal branch

$51 \%, 30 \%$, and $28 \%$, respectively (2). These trends of the outcomes across the time are explained by improvements in obstetric, pediatric and intensive care but, also, by the evolution of endovascular techniques and materials. At the beginning of the endovascular treatment of VOGMs only glue (n-butyl cyanoacrylate) and coils were available as embolic materials. Glue was the standard embolic liquid for VOGM for a long time. However, embolization with glue is challenging due to its adhesive characteristic that limits a precise control of the material during injections. Coiling have also been done in the treatment of VOGMs, but coiling is feasible for only some mural shunts where coils can be safely placed. More recently the ethylene vinyl alcohol copolymer $(E V O H)$ has substitute glue for embolization of diverse arteriovenous malformations $(4,5)$. Compared to glue, EVOH allows for a more controlled and more precise embolization. In addition to $\mathrm{EVOH}$, the new DLB have the property to be used to inject EVOH during a temporary occlusion of the balloon. The occlusion of the balloon blocks the flow protecting patients of an inadvertent EVOH migration 


\section{CURATIVE EMBOLIZATION OF A VEIN OF GALEN MALFORMATION WITH A DUAL-LUMEN BALLOON AND ETHYLENE VINYL ALCOHOL COPOLYMER}
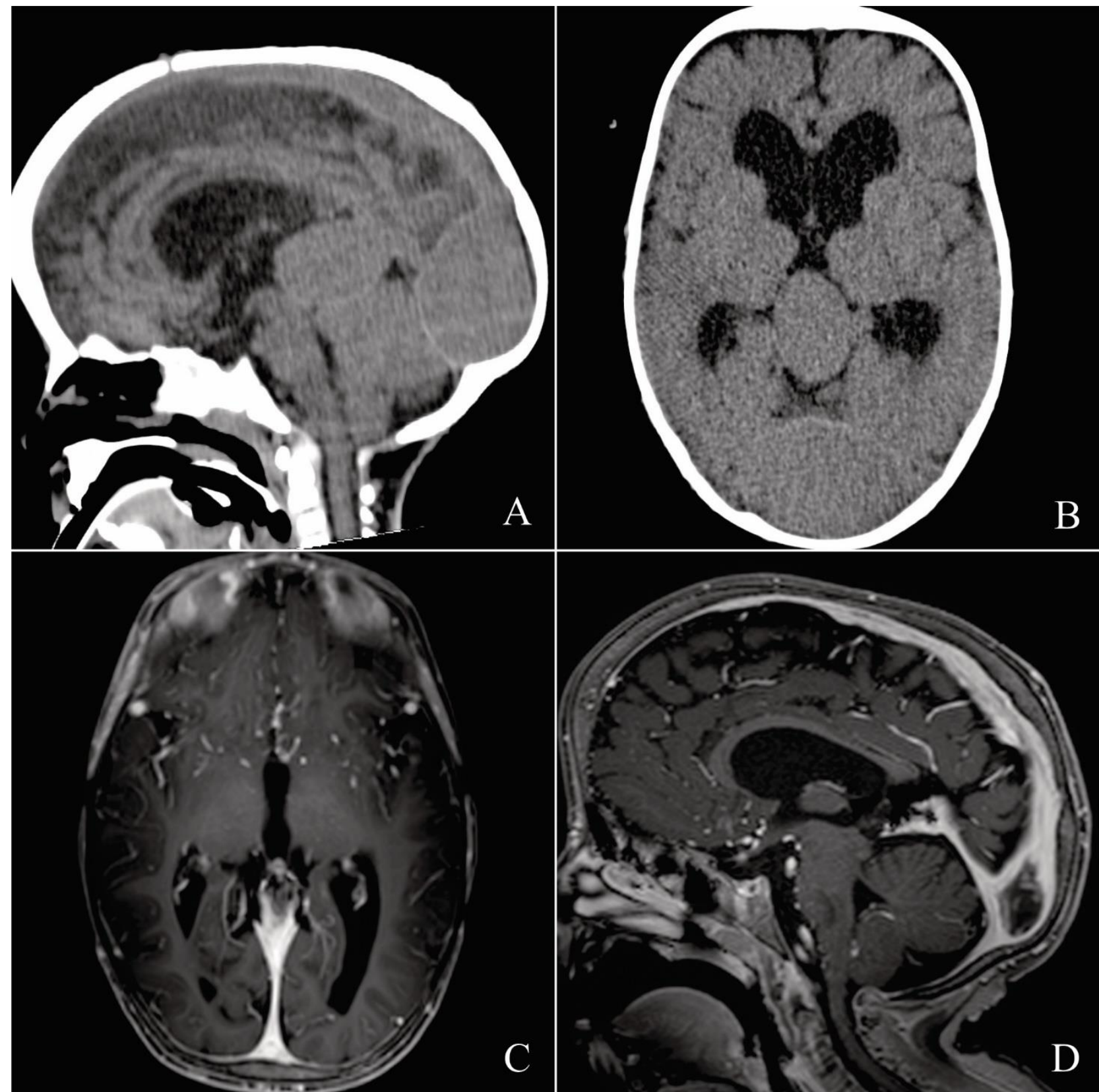

Figure 3 - Computed tomography scan of the brain at admission, sagittal view (A), and axial view (A) showing a dilated vein of Markowski and a dilated torcula. Magnetic resonance image of the brain 12 months after treatment axial view (C), and sagittal view (D) showing absence of the vein of Markowski and a reduction in size of the torcula

through the shunt to the veins and lungs. The balloon also prevents proximal reflow of EVOH reducing the risk ischemic complications caused by entrance of EVOH in normal perforators (6). In the present case we demonstrated two strategies of transarterial (TA) embolization of a mural type VOGM presenting with two direct shunts. The first shunt was embolized using a cast of coils to reduce the flow through the shunt, following by an EVOH injection which resulted in the complete occlusion of that shunt (Figure 1). The second shunt was embolized also with EVOH but through a DLB (Figure 2). In the second embolization a precise cast of EVOH could be created resulting in a complete occlusion of the
VOGM. This case demonstrated the advantages of DLB compared to other embolization techniques.

Regarding the embolization strategies, both transvenous (TV) or TA accesses have been used in the treatment of VOGM. In general, the TV strategy is technically easier compared to the TA approach. As VOGMs usually present enlarged venous routes, a TV coiling of the vein of Markowski is feasible in the treatment of some mural types of VOGM. However, for choroidal types or mixed types of VOGM, the TV carries a higher hemorrhagic risk compared to the TA strategy. Therefore, the TA has become the first line strategy in the treatment of VOGM. In TA approach only the shunts 


\section{CURATIVE EMBOLIZATION OF A VEIN OF GALEN MALFORMATION WITH A DUAL-LUMEN BALLOON AND ETHYLENE VINYL ALCOHOL COPOLYMER}

are occluded avoiding occlusion of draining veins, which could increase the risk of hemorrhagic complication. However, TA has limitations that includes the risks of distal migration of embolic material causing venous thrombosis, or pulmonary embolism, or proximal arterial reflux of the embolic material causing infarction. Glue only, or even EVHO only, injected through microcatheters in high flow mural shunts are associated with the risk of distal migration of the material to the veins and lungs, or with the risk ischemic stroke. In the present case, the use of coils in the first session resulted in a reduction of the flow through the shunt, so EVHO could be injected under a slow flow. However, compared to coiling the DLB allowed for a more precise embolization because the artery remained blocked and protected proximally. Therefore, a more controllable embolization could be performed by a slowly injection of EVOH until it reached the initial portion of the vein (Figure 2). This technique has limitations. First, the overinflation of the DLB can cause an arterial rupture. Second, DLB cannot be navigated through small and tortuous arteries which limits its use. Perhaps the new low profile DLB can be navigated and inflated in small and tortuous arteries which will expand the indication of this technique during the treatment of vOGMs.

\section{CONCLUSION}

Embolization of VOGMs using ethylene vinyl alcohol copolymer through a DLB is feasible. Large studies are necessary to assess the safety and the impact of this technique in the curative rates of VOGMs.

\section{REFERENCES}

1. Yan J, Gopaul R, Wen J, Li XS , Tang JF. The natural progression of VGAMs and the need for urgent medical attention: a systematic review and meta-analysis. J Neurointerv Surg 2017;9:564-570

2. Yan J, Wen J, Gopaul R, Zhang CY, Xiao SW. Outcome and complications of endovascular embolization for vein of Galen malformations: a systematic review and metaanalysis. J Neurosurg. 2015;123:872-90

3. Bhatia K, Mendes Pereira V, Krings T, Ter Brugge $K$, Kortman $H$, Dirks $P$, Armstrong D, Shroff M, Muthusami P. Factors Contributing to Major Neurological Complications From Vein of Galen Malformation Embolization. JAMA Neurol 2020;77:992-999

4. Kim DJ, Suh DC, Kim BM, Kim DI. Adjuvant Coil Assisted Glue Embolization of Vein Of Galen Aneurysmal Malformation in Pediatric Patients. Neurointervention 2018;13:41-47

5. Orlov K, Gorbatykh A, Berestov V, Shayakhmetov T, Kislitsin D, Seleznev $P$, Strelnikov N. Superselective transvenous embolization with Onyx and n-BCA for vein of Galen aneurysmal malformations with restricted transarterial access: safety, efficacy, and technical aspects. Childs Nerv Syst 2017;33:2003-2010

6. Pop R, Manisor M, Wolff V, Kehrli P, Marescaux C, Beaujeux R. Flow control using Scepter ${ }^{\mathrm{TM}}$ balloons for Onyx embolization of a vein of Galen aneurysmal malformation. Childs Nerv Syst. 2015;31:135-40 